\title{
Secreted form of EphA7 in lung cancer
}

\author{
MASARU TSUBOI ${ }^{1}$, HIROKI MORI ${ }^{1}$, TOMOYASU BUNAI ${ }^{1}$, SHINJI KAGEYAMA ${ }^{1}$, MASAYA SUZUKI ${ }^{1}$, \\ KOJI OKUDELA ${ }^{1}$, KAZUYA TAKAMOCHI ${ }^{2}$, HIROSHI OGAWA ${ }^{3}$, HIROSHI NIWA ${ }^{4}$, \\ KAZUYA SHINMURA $^{1}$ and HARUHIKO SUGIMURA ${ }^{1}$
}

\author{
Departments of ${ }^{1}$ Pathology and ${ }^{2}$ Respiratory Surgery, Hamamatsu University School of Medicine, \\ 1-20-1 Handayama, Higashi-ward, Hamamatsu 431-3192; Departments of ${ }^{3}$ Pathology and ${ }^{4}$ Respiratory Surgery, \\ Seirei Mikatahara Hospital, 3453 Mikataharacho, Kita-ward, Hamamatsu 423-8558, Japan
}

Received September 25, 2009; Accepted November 18, 2009

DOI: 10.3892/ijo_00000539

\begin{abstract}
EPHA7 is a member of the EPHA family of receptor kinases, among which several members are known to be involved in human lung carcinogenesis. We report here a novel spliced variant, the so-called secreted form of EPHA7, recently reported in malignant lymphoma, in human lung cancer cell lines and primary lung cancer. In contrast to the EPHA7 down-regulation in colorectal cancer by promoter hypermethylation, EPHA7 is expressed at a substantial level in most human lung cancers and the secreted form of EPHA7 mRNA was found in a fraction of primary lung cancer tissues, lung cancer cell lines, and immortalized bronchogenic epithelial cell lines. Interestingly, the secreted form of EPHA7 message was predominantly detected in non-adeno type lung carcinoma. The mechanistic role of the secreted form of EPHA7 in human lung carcinogenesis is not clear, but the presence of this form could distinctly exclude adenocarcinoma of the lung from the other categories, i.e., squamous cell carcinoma, small cell carcinoma and large cell carcinoma, which have strong association with smoking. This is the first study to detect the secreted form of EPHA7 in human epithelial tissues. EPHA7 warrants further investigation to determine its possible involvement in smoking related lung carcinogenesis.
\end{abstract}

\section{Introduction}

The erythropoietin producing hepatocellular carcinoma (EPH) family of receptor tyrosine kinases constitutes the RTK subfamily and members of this family are divided into EPHAs and EPHBs. EPHAs are typically bound to Ephrin(EFN)As, which are anchored to the cell membrane via a glycosylphosphatidylinositol anchor. EPHBs are typically bound to EFNBs, which have a transmenbrane domain (1). These molecules

Correspondence to: Dr Haruhiko Sugimura, First Department of Pathology, Hamamatsu University School of Medicine, 1-20-1 Handayama, Higashi-ward, Hamamatsu 431-3192, Japan

E-mail: hsugimur@hama-med.ac.jp

Key words: EPHA7, lung cancer take bidirectional signal pathways, EFN to EPH forward signal and EPH to EFN reverse signal, and these pathways are involved in many physiological and pathological conditions (2). Members of this family play critical roles in many facets of cancer biology, from initiation to metastasis and also invasion (3-6). Almost all the human cancers have been reported to be associated with some of the EPH-EFN pathways (7-11). EPHA family genes, especially EPHA3 and EPHA5, are among the genes which often mutate in human lung cancer, as demonstrated by a recent extensive large scale coding sequence analysis (12). EPHA7 is a member of the EPHA family, but it has been investigated in only a few human lung cancers. The recent finding of its downregulation by promoter methylation and a possible tumor suppressing effect in several types of human cancer $(8,13,14)$ prompted us to investigate the expression status of EPHA7 in human lung cancer, to assess the possibility of a tumor suppressor role in lung carcinogenesis. On the other hand, the locus of EPHA7 is near the breakpoint of $\mathrm{t}(3 ; 6)$ in renal cell carcinoma (15). Considering the recent discovery of various translocations in solid tumors $(16,17)$, especially in lung carcinoma (18-20), we also anticipated translocation involving this gene locus in lung cancer.

\section{Materials and methods}

Cell lines. Forty cell lines, consisting of 3 normal lung cells (SAEC, 16HBET14o- and WI-38), 10 lung adenocarcinomas (H358, H820, H2087, A549, HLC-1, RERF-LC-MS, RERFLC-KJ, LC-2/ad, VMRC-LCD and PC-3), one lung squamous cell carcinoma (ABC-1), four lung large cell carcinomas (H460, H1299, LU65 and PC-13), five lung small cell carcinomas (H526, H1688, TKB-2, Lu-130 and Lu-135), three colon adenocarcinomas (HCT116, HT29 and DLD-1), eight gastric adenocarcinomas (AGS, HSC-39, KATO3, MKN-1, MKN-28, MKN-45, MKN-74 and TMK-1) and four esophageal squamous cell carcinomas (A431, HSC-2, HSC-3 and HSC-4), were used in this study. The 16HBE14o- cell line (Simian virus 40-transformed human bronchial epithelial cells) was a gift from Dr D.C. Gruenert (California Pacific Medical Center Research Institute, San Francisco, CA, USA) via Dr T. Kaneko (Department of Internal Medicine, Yokohama City University, School of Medicine, Yokohama, 
Japan) (21). The Lu-130, Lu-135 and PC-13 cell lines were gifts from Dr Y. Dobashi (Jichi Medical University, Omiya Medical Center Hospital, Omiya, Japan). A549, ABC-1, H460, LC-2/ad, VMRC-LCD, RERF-LC-MS, REAF-LCKJ and TKB-2 were gifts from Dr T. Niki (Jichi Medical University, Shimotsuke, Japan). TMK-1 was a gift from the Department of Genetics, National Cancer Center (Tokyo, Japan). SAEC was purchased from Clontech (San Diego, CA). WI-38, H358, H820, H2087, H1299, H526, H1688, HCT116, HT-29, DLD-1 and AGS were obtained from ATCC (Manassas, VA, USA). PC-3, LU65, KATO3, MKN-1, MKN-28, MKN-45, MKN-74, A431, HSC-2, HSC-3 and HSC-4 were obtained from the Health Science Research Resources Bank (Osaka, Japan). HLC-1 was obtained from RIKEN Cell Bank (Ibaraki, Japan).

Clinical samples. The subjects were selected from among patients of Hamamatsu University School of Medicine and Mikatahara Seirei General Hospital. Written informed consent to participate in this study was obtained and the entire study design was approved by the Institutional Review Boards (IRB) of Hamamatsu University School of Medicine (18-4,18-5) and Mikatahara Seirei General Hospital. Lifestyle information such as smoking habits was obtained by professional interviewers. Histopathological classification was performed according to the WHO classification (2004) (22). Stages of the clinical samples according to the TNM classification system (http://www.uicc. org/) are shown in Table I.

3'-Rapid amplification of cDNA end. 3'-RACE was performed with the 3' RACE System for Rapid Amplification of cDNA Ends (Invitrogen). Briefly, the first strand cDNA was reverse transcribed from $1 \mu \mathrm{g}$ of total RNA using SuperScript II RT (Invitrogen) and the adapter primer; $1 \mu \mathrm{l}$ of the first strand cDNA was then amplified using an EPHA7 gene-specific forward primer (EphA7 RACE 5'-CACCATACGTTGCATG CACA-3') and the Universal Amplification Primer. In the polymerase chain reactions, after initial denaturation at $95^{\circ} \mathrm{C}$ for $15 \mathrm{~min}, 35$ cycles of denaturation at $95^{\circ} \mathrm{C}$ for $30 \mathrm{sec}$, annealing at $58^{\circ} \mathrm{C}$ for $30 \mathrm{sec}$, and elongation at $72^{\circ} \mathrm{C}$ for $45 \mathrm{sec}$ were used, followed by a final elongation step at $72^{\circ} \mathrm{C}$ for $5 \mathrm{~min}$.

Sequencing of the RACE product and validating sequencing of the secreted form of EPHA7 (EPHA7-S). We sequenced the band of $\sim 600$ bp generated by the RACE procedure with an EPHA7 gene-specific forward sequencing primer, and identified it as EPHA7-S. We searched for EPHA7-S in lung cancer cell lines by sequencing the cDNAs synthesized from mRNAs. Gene-specific primer pairs were designed to cover the region from exon 4 to intron 5 of EPHA7. Primers for PCR were 5'-CATCTGACCCACCATACGTTGC-3' (EPHA7 exon 4) and 5'-GCTGGAAGAATCAAGCTCTGTG-3' (EPHA7 intron 5). PCR was carried out in reaction mixtures containing cDNA, 1X HotStar Taq buffer, $0.25 \mathrm{mmol} / 1$ deoxynucleotide triphosphate mixture, $0.05 \mathrm{U}$ of HotStar Taq (Qiagen, Dusseldorf, Germany), and $0.5 \mathrm{mmol} / \mathrm{l}$ of forward and reverse primers in a volume of $20 \mu \mathrm{l}$. PCR cycling parameters were one cycle of $95^{\circ} \mathrm{C}$ for $15 \mathrm{~min}$; 40 cycles of $95^{\circ} \mathrm{C}$ for $30 \mathrm{sec}, 58^{\circ} \mathrm{C}$ for $30 \mathrm{sec}$, and $75^{\circ} \mathrm{C}$ for $45 \mathrm{sec}$;
Table I. Clinicopathological characteristics of the patients.

No of patients, $\mathrm{N}$ 73

Average age (range), years

Gender, n (\%)

Male

Female

Histology, n (\%)

Adenocarcinoma

Squamous cell carcinoma

Small cell carcinoma

Large cell carcinoma

Brinkman index, $\mathrm{n}(\%)$

$\mathrm{BI}=0$

$0<\mathrm{BI} \leq 400$

$400<$ BI

Unknown

TNM stage, n (\%)

I

II

III

IV

Unknown

followed by one cycle of $72^{\circ} \mathrm{C}$ for $5 \mathrm{~min}$. The PCR products were purified with a PCR purification kit (Qiagen) and directly sequenced with a Big Dye Terminator Cycle Sequencing Reaction Kit and the ABI 3100 Genetic Analyzer (Applied Biosystems Incorporated, Tokyo, Japan). Sequencing reactions were done in both forward and reverse directions with two primers for PCR.

Statistical analysis. $\chi^{2}$ analysis and the Cochran-Armitage trend test were conducted to compare EPHA7-S expression with various clinical features (sex, smoking history, histological type, and TNM stages). A $\mathrm{p}<0.05$ was considered significant. Statistical analyses were performed using the SAS (Statistical Analysis System) program (SAS Institute Japan, Tokyo, Japan).

\section{Results}

Detection of EPHA7-S in cell lines. First, we attempted to identify a downstream sequence, expecting a fusion partner of EPHA7. We adopted the 3'-RACE method using the primer corresponding to exon 4 of EPHA7 and the RACE specific 3 ' primer for the RNAs from PC-13 and H82 cell lines, both of which express EPHA7 (data not shown). The RERF-LC-MS cell line, which has no EPHA7 expression, was used as a negative control. By the 3'-RACE method, we detected amplified fragments in both PC-13 and H82 (Fig. 1a). 
$\mathbf{a}$

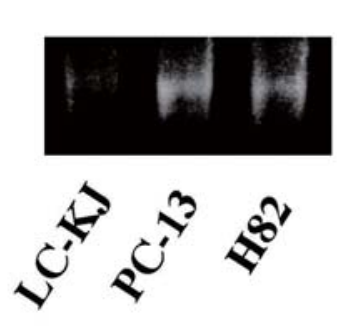

b

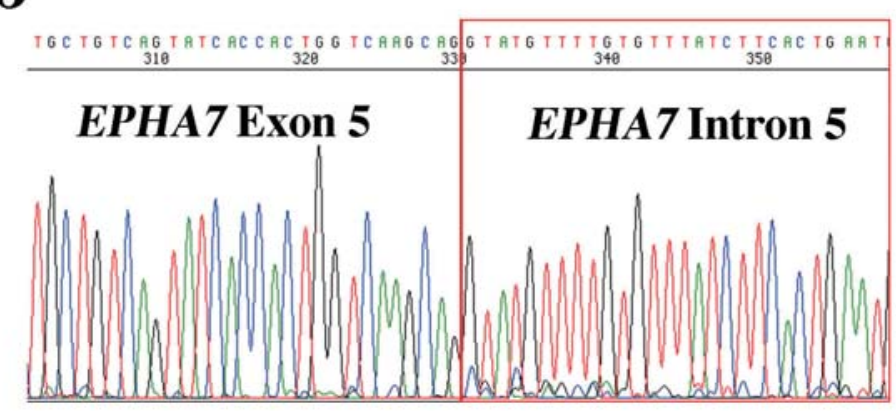

c

Exon 5

\begin{tabular}{|c|c|c|c|c|c|c|c|c|c|c|c|c|c|c|c|c|c|}
\hline \multicolumn{9}{|c|}{ EXOII } & & & & & & & & & \multirow[b]{2}{*}{ EPHA7-FL } \\
\hline $\mathbf{A}$ & V & $\mathrm{S}$ & I & $\mathbf{T}$ & $\mathbf{T}$ & G & $\mathbf{Q}$ & $\mathbf{A}$ & & & & & & & & & \\
\hline & & & & & & & & & & & & & & & & & \\
\hline $\mathbf{A}$ & $\mathbf{V}$ & $\mathbf{S}$ & I & $\mathbf{T}$ & $\mathbf{T}$ & G & $\mathbf{Q}$ & $\mathbf{A}$ & G & $\mathbf{M}$ & $\mathbf{F}$ & $\mathrm{C}$ & $\mathbf{V}$ & $\mathbf{Y}$ & $\mathbf{L}$ & H Stop- & - EPHA7-S \\
\hline
\end{tabular}

d

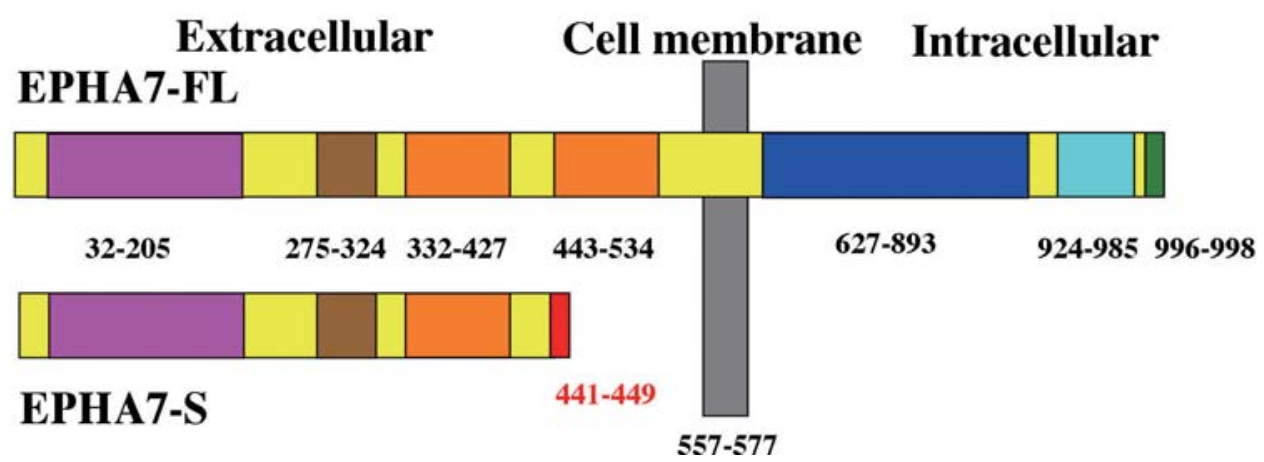

Figure 1. Detection of the secreted form of EPHA7 (EPHA7-S) in lung cancer cell lines. (a) 3'-RACE method showing amplification of EPHA7-S in PC-13 and H82 but not in RERF-LC-KJ. (b) Sequencing analysis of the EPHA7-S gene transcript of PC-13. The red box indicates intron 5 of the EPHA7 gene. (c) Nucleotide sequence and predicted amino acid sequence of full-length EPHA7 (EPHA7-FL) at the end of exon 5 and for the EPHA7-S based on sequencing of a cDNA from the PC-13 cell line. Shaded region is exon 5 of the EPHA7 gene. (d) Schematic representation of EPHA7-FL and EPHA7-S. 32-205, Ephrin receptor ligand binding domain; 275-324, TNF receptor domain; 332-427 and 443-534, Fibronectin type 3 domain; 557-577, transmenbrane domain; 627-893, tyrosine kinase domain; 924-985, SAM domain; 996-998, PDZ binding motif; 441-449, intron 5 (EPHA7-S).

Sequencing analysis of these fragments disclosed that they were not fusion partners, while the sequence derived from EPHA7 itself had its intron 5 (Fig. 1b and c). DNase treatment and the cDNA minus negative control excluded the possibility of mis-amplification of the genomic sequence. This cDNA contained the exon 5 sequence directed to the intron 5 sequence of EPHA7 (Fig. 1c), and it had a stop codon after coding 8 amino acids, generating the structure lacking the transmembrane domain of the authentic EPHA7 (Fig. 1d). Given these structural features, we consider this form to be the human counterpart of EphA7-S reported in murine lymphocytes by Dawson et al (23). In addition, they demonstrated human tonsillar lymphocytes to also express an EPHA7-S protein of consistent size by Western blotting, though the exact message was not shown (23). This structure lacks a cytoplasmic domain, which would include a kinase domain, indicating that the product would be secreted outside the cells.

EPHA7-S expression in lung cell lines. We examined cell lines, including those of lung cancers, gastrointestinal cancers and immortalized bronchial epithelium, for detection of EPHA7-S. We used the primers in the EPHA7 exon 4 (forward) and intron 5 (reverse) for reverse transcription PCR. This primer set discriminates the contaminated genomic amplified product (1677 bp) from the target product (475 bp) (Fig. 2a). EPHA7-S was detected mainly in lung cancer cell lines, rarely in those from other organs $(16 / 21$ in lung cancer cell lines vs. 1/15 gastrointestinal cancer cell lines) (Fig. 2b and Table II), that is, EPHA7-S is a variant occurring mainly in lung cancer cells. 
a

\section{EPHA7 genomic DNA \\ EX4 INT4 EX5 INT5 EX6 \\ F-primer R-primer 1677 bp \\ EPHA7-FL CDNA \\ EX4 EX5 EX6}

F-Primer
EPHA7-S cDNA
EX4 EX5 INT5
F-primer R-primer

b

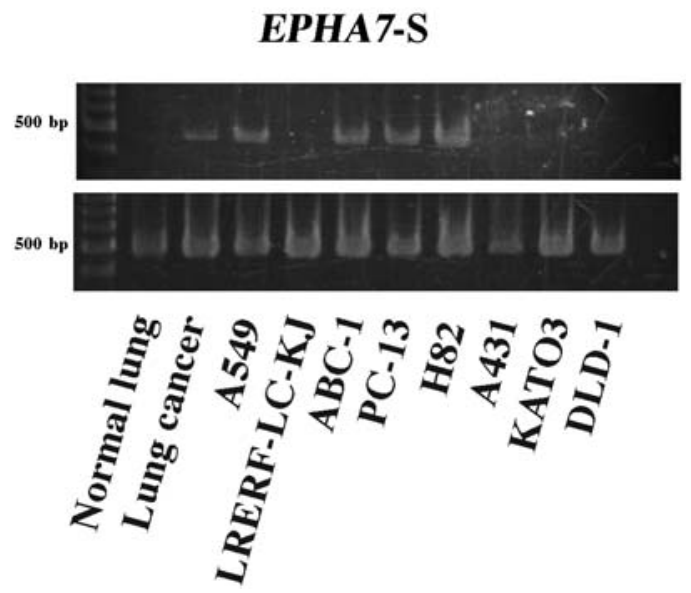

Figure 2. Secreted form of EPHA7 (EPHA7-S) in various cell lines. (a) PCR primer design and the structure of EPHA7-S. The amplified structure of genomic EPHA7 (1677 bp) is shown above, EPHA7-FL cDNA (no amplification) is the middle, and EPHA7-S cDNA (475 bp) below. (b) EPHA7-S cDNA (475 bp) was detected in some of these cell lines. The bands in the upper panel are EPHA7-S and those in the lower panel sare GAPDH. The sources of the normal lung and lung cancer lanes are clinical samples from patient no. 15 (squamous cell carcinoma and the corresponding non-tumor lung portion). A549, LRERF-LC-KJ, ABC-1, PC13 and $\mathrm{H} 82$ are lung cancer cell lines, A431 is an esophageal cancer cell line, KATO3 is a gastric cancer cell line, and DLD-1 is a colon cancer cell line. The left-most lane indicates size markers, a 100-bp DNA ladder.

Some clinical lung cancer specimens express EPHA7-S. The tendency for EPHA7 to be expressed by lung cancer cell lines prompted us to look for it in primary lung cancer tissues. A substantial proportion of human primary lung cancers expressed EPHA7-S; 2 out of 50 cases with lung adenocarcinoma and 8 of 23 cases with non-adenocarcinoma of the lung. Among the non-adenocarcinoma cases, 6 of 17 squamous cell carcinoma cases, one of the two cases with small cell carcinoma, and one of four large cell carcinoma cases were positive for EPHA7-S (Table III). In 2 cases, EPHA7-S was detected in non-tumor lung tissue adjacent to the lung cancer (data not shown).

The prevalence of EPHA7-S was significantly greater in non-adenocarcinoma than in adenocarcinoma (Table IV). No
Table II. Secreted form of EPHA7 (EPHA7-S) in various cell lines.

\begin{tabular}{|c|c|c|}
\hline Material & & EphA7-S \\
\hline 16HBE14o- & $\begin{array}{l}\text { Normal human bronchial } \\
\text { epithelium }\end{array}$ & + \\
\hline SAEC & Small airway epithelial & + \\
\hline WI-38 & Lung fibroblast & - \\
\hline H358 & Lung adenocarcinoma & + \\
\hline H820 & Lung adenocarcinoma & + \\
\hline H2087 & Lung adenocarcinoma & - \\
\hline A549 & Lung adenocarcinoma & + \\
\hline HLC-1 & Lung adenocarcinoma & + \\
\hline RERF-LC-MS & Lung adenocarcinoma & + \\
\hline LC-2/ad & Lung adenocarcinoma & - \\
\hline VMRC-LCD & Lung adenocarcinoma & + \\
\hline PC-3 & Lung adenocarcinoma & + \\
\hline RERF-LC-KJ & Lung adenocarcinoma & - \\
\hline $\mathrm{ABC}-1$ & Lung squamous cell carcinoma & + \\
\hline H460 & Lung large cell carcinoma & - \\
\hline H1299 & Lung large cell carcinoma & + \\
\hline LU65 & Lung large cell carcinoma & + \\
\hline PC-13 & Lung large cell carcinoma & + \\
\hline H82 & Lung small cell carcinoma & + \\
\hline H526 & Lung small cell carcinoma & + \\
\hline H1688 & Lung small cell carcinoma & + \\
\hline Lu-130 & Lung small cell carcinoma & + \\
\hline Lu-135 & Lung small cell carcinoma & + \\
\hline TKB-2 & Lung small cell carcinoma & - \\
\hline DLD-1 & Colon adenocarcinoma & - \\
\hline НCТ-116 & Colon adenocarcinoma & - \\
\hline HT29 & Colon adenocarcinoma & - \\
\hline AGS & Gastric adenocarcinoma & - \\
\hline HSC-39 & Gastric adenocarcinoma & + \\
\hline KATO3 & Gastric adenocarcinoma & - \\
\hline MKN-1 & Gastric adenocarcinoma & - \\
\hline MKN28 & Gastric adenocarcinoma & - \\
\hline MKN-45 & Gastric adenocarcinoma & - \\
\hline MKN-74 & Gastric adenocarcinoma & - \\
\hline TMK-1 & Gastric adenocarcinoma & - \\
\hline A431 & $\begin{array}{l}\text { Esophageal squamous cell } \\
\text { carcinoma }\end{array}$ & - \\
\hline HSC-2 & $\begin{array}{l}\text { Esophageal squamous cell } \\
\text { carcinoma }\end{array}$ & - \\
\hline HSC-3 & $\begin{array}{l}\text { Esophageal squamous cell } \\
\text { carcinoma }\end{array}$ & - \\
\hline HSC-4 & $\begin{array}{l}\text { Esophageal squamous cell } \\
\text { carcinoma }\end{array}$ & - \\
\hline
\end{tabular}

+, EPHA7-S detectable, -, not detectable. 
Table III. Clinicopathological characteristics of 10 patients with EPHA7-S-positive lung cancer.

\begin{tabular}{lrrcrr}
\hline Patient no. & Sex & Age & Histology & Stage & BI \\
\hline 9 & F & 77 & SCC & $3 \mathrm{~b}$ & 0 \\
15 & M & 62 & SQCC & $3 \mathrm{a}$ & 1260 \\
18 & M & 61 & SQCC & $2 \mathrm{~b}$ & 1200 \\
24 & M & 69 & SQCC & $1 \mathrm{a}$ & 740 \\
31 & M & 39 & SQCC & $1 \mathrm{~b}$ & 920 \\
34 & M & 66 & LCC (LCNEC) & $1 \mathrm{~b}$ & 1350 \\
42 & M & 65 & ADC & $1 \mathrm{a}$ & 1350 \\
47 & F & 82 & ADC & $1 \mathrm{a}$ & 0 \\
55 & F & 54 & SQCC & $3 \mathrm{~b}$ & 720 \\
63 & F & 67 & SQCC & $1 \mathrm{a}$ & 0 \\
\hline
\end{tabular}

ADC, adenocarcinoma; LCC, large cell carcinoma; LCNEC, large cell neuroendocrine carcinoma; SCC, small cell carcinoma; SQCC, squamous cell carcinoma; BI, Brinkman index.

Table IV. Associations between EPHA7-S and clinicopathological features.

\begin{tabular}{|c|c|c|c|}
\hline & EPHA7-S ${ }^{+}$ & EPHA7-S- & P-value \\
\hline \multicolumn{4}{|l|}{ Gender } \\
\hline Male & 6 & 35 & $0.7924^{\mathrm{a}}$ \\
\hline Female & 4 & 28 & \\
\hline \multicolumn{4}{|l|}{ Histology } \\
\hline Adenocarcinoma & 2 & 48 & $0.0004^{\mathrm{a}}$ \\
\hline Other carcinomas & 8 & 15 & \\
\hline \multicolumn{4}{|l|}{ Brinkman index } \\
\hline $0 \leq \mathrm{BI}<400$ & 3 & 25 & $0.557^{\mathrm{a}}$ \\
\hline $400 \leq \mathrm{BI}$ & 7 & 29 & \\
\hline \multicolumn{4}{|l|}{ TNM stage } \\
\hline I & 6 & 43 & $0.3548^{\mathrm{b}}$ \\
\hline II & 1 & 4 & \\
\hline III & 3 & 10 & \\
\hline IV & 0 & 3 & \\
\hline
\end{tabular}

${ }^{\mathrm{a}} \chi^{2}$ test. ${ }^{\mathrm{b}}$ Cochran-Armitage trend test.

other clinicopathological factors were related to EPHA7-S expression.

\section{Discussion}

While pursuing the possibility of EPHA7 fusion partners, we incidentally isolated EPHA7-S in lung cancer cell lines and tissues. Intriguingly, EPHA7-S tends to be expressed in lung but not other cancer cell lines. Furthermore, this EPHA7-S expression in lung cancers was seen mainly in non-adenocarcinomas. These findings may facilitate diagnosing of the primary site of such cancers, especially non-adenocarcinoma types.

Lung carcinogenesis probably constitutes heterogeneous steps according to its histological subtype and/or environmental effects such as smoking. Classically, non-adenocarcinomas are believed to have a stronger association with smoking than adenocarcinoma of the lung (24), such that the greater representation of EPHA7-S in non-adenocarcinoma than adenocarcinoma may suggest this difference to reflect EPHA7-S involvement in smoking related carcinogenesis. The amount of tobacco did not, however, correlate with the EPHA7-S expressions in the tumors of our subjects. Thus, the significance of this tendency must be carefully interpreted and further accumulation of cases is warranted. Recently, expression of EPHA2, another EPHA family member, was reported to be associated with smoking history (25). EPHA7-S is possibly another member of the EPH family of genes related to all form of smoking. The prevalences of EPHA7-S in lung cancer cell lines of different histological origin did not, however, differ between adenocarcinomas and non-adenocarcinomas. Again, the significance of the apparent difference in primary lung tumors according to subtype remains an open question. There is only one report documenting EPHA7-S expression in human tumors. Dawson et al reported EPHA7-S in germinal center lymphocytes (23). They speculated that hypermethylation of the EPHA7 promoter and the secreted form of EphA7 interacted in lymphomatogenesis. In fact, the finding of frequent promoter methylation in EPHA7 in colorectal cancer raised the possibility that EPHA7 is one of the tumor suppressor genes inactivated in colorectal carcinogenesis (8). The EPHA7 expression profile in human lung cancers was quite different from those in colorectal, gastric, prostate, and brain cancers $(8,13,14,26)$. EPHA7 was expressed mainly in the tumor portion (data not shown). In this context, EPHA7 may be an oncogene rather than a suppressor gene in lung carcinogenesis. A larger clinical study is needed to corroborate this view of EPHA7 as an oncogene. This paradoxical situation (oncogene in one and suppressor gene in an other organ) has been described in several organs and settings in which the $E P H$ family of genes play roles (27). EPHA7-S is also overexpressed in some lung cancers, meaning that the role of EPHA7-S in lung cancer may not be consistent with that assumed in lymphoma, as previously reported (23). We detected EPHA7-S in two nontumor lung tissues. We do not know the exact reason, but environmental stress may induce various forms of aberrant splicing (28). Since we also detected EPHA7 in SAEC and 16HBE140- cell lines, both of which are from non-tumorigenic bronchogenic epithelium, the same microenvironment as in lung cancer tissue may exist in the non-tumor portion of the lung. In addition, there was an exception: HSC-39, a gastric cancer cell line, expresses EPHA7-S. The biological and pathological situations triggering EPHA7-S expression clearly require further study. In conclusion, we have, for the first time, described the secreted form of EPHA7 in a subset of human lung cancers. 


\section{Acknowledgments}

This work is supported in part by a Grant-in-Aid from the Ministry of Education, Culture, Sports, Science and Technology (20014007), from the Ministry of Health, Labor, and Welfare (19-19), and the Smoking Research Foundation.

\section{References}

1. Eph nomenclature and committee: unified nomenclature for Eph family receptors and their ligands, the ephrins. Eph Nomenclature Committee. Cell 90: 403-404, 1997.

2. Pasquale EB: Eph-ephrin bidirectional signaling in physiology and disease. Cell 133: 38-52, 2008.

3. Nakamoto $M$ and Bergemann AD: Diverse roles for the Eph family of receptor tyrosine kinases in carcinogenesis. Microsc Res Tech 59: 58-67, 2002.

4. Noren NK, Lu M, Freeman AL, Koolpe M and Pasquale EB: Interplay between EphB4 on tumor cells and vascular ephrinB2 regulates tumor growth. Proc Natl Acad Sci USA 101: 5583-5588, 2004.

5. Campbell TN, Attwell S, Arcellana-Panlilio M and Robbins SM: Ephrin A5 expression promotes invasion and transformation of murine fibroblasts. Biochem Biophys Res Commun 350: 623-628, 2006.

6. Tanaka M, Sasaki K, Kamata R and Sakai R: The C-terminus of ephrin-B1 regulates metalloproteinase secretion and invasion of cancer cells. J Cell Sci 120: 2179-2189, 2007.

7. Nakamura R, Kataoka H, Sato N, et al: EPHA2/EFNA1 expression in human gastric cancer. Cancer Sci 96: 42-47, 2005.

8. Wang J, Kataoka H, Suzuki M, et al: Downregulation of EphA7 by hypermethylation in colorectal cancer. Oncogene 24: 5637-5647, 2005.

9. Taddei ML, Parri M, Angelucci A, et al: Kinase-dependent and -independent roles of EphA2 in the regulation of prostate cancer invasion and metastasis. Am J Pathol 174: 1492-1503, 2009.

10. Kataoka H, Igarashi H, Kanamori M, et al: Correlation of EPHA2 overexpression with high microvessel count in human primary colorectal cancer. Cancer Sci 95: 136-141, 2004.

11. Kataoka H, Tanaka M, Kanamori M, et al: Expression profile of EFNB1, EFNB2, two ligands of EPHB2 in human gastric cancer. J Cancer Res Clin Oncol 128: 343-348, 2002.

12. Ding L, Getz G, Wheeler DA, et al: Somatic mutations affect key pathways in lung adenocarcinoma. Nature 455: 1069-1075, 2008.
13. Guan M, Xu C, Zhang F and Ye C: Aberrant methylation of EphA7 in human prostate cancer and its relation to clinicopathologic features. Int J Cancer 124: 88-94, 2009.

14. Wang J, Li G, Ma H, et al: Differential expression of EphA7 receptor tyrosine kinase in gastric carcinoma. Hum Pathol 38: 1649-1656, 2007.

15. Foster RE, Abdulrahman M, Morris MR, et al: Characterization of a 3;6 translocation associated with renal cell carcinoma. Genes Chromosomes Cancer 46: 311-317, 2007.

16. Tognon C, Knezevich SR, Huntsman D, et al: Expression of the ETV6-NTRK3 gene fusion as a primary event in human secretory breast carcinoma. Cancer Cell 2: 367-376, 2002.

17. Tomlins SA, Laxman B, Dhanasekaran SM, et al: Distinct classes of chromosomal rearrangements create oncogenic ETS gene fusions in prostate cancer. Nature 448: 595-599, 2007.

18. Soda M, Choi YL, Enomoto M, et al: Identification of the transforming EML4-ALK fusion gene in non-small-cell lung cancer. Nature 448: 561-566, 2007.

19. Takeuchi K, Choi YL, Togashi Y, et al: KIF5B-ALK, a novel fusion oncokinase identified by an immunohistochemistry-based diagnostic system for ALK-positive lung cancer. Clin Cancer Res 15: 3143-3149, 2009.

20. Rikova K, Guo A, Zeng Q, et al: Global survey of phosphotyrosine signaling identifies oncogenic kinases in lung cancer. Cell 131: 1190-1203, 2007.

21. Okudela K, Suzuki M, Kageyama S, et al: PIK3CA mutation and amplification in human lung cancer. Pathol Int 57: 664-671, 2007.

22. Kleihues Pand L and Sobin H: (eds.) Tumours of the lung, pleura, thymus and heart. IARC Press, Lyon, 2004.

23. Dawson DW, Hong JS, Shen RR, et al: Global DNA methylation profiling reveals silencing of a secreted form of Epha7 in mouse and human germinal center B-cell lymphomas. Oncogene 26: 4243-4252, 2007.

24. Kreyberg L: Relationship of different histological lung tumour groups to tobacco smoking. Br J Cancer 15: 51-53, 1961.

25. Brannan JM, Dong W, Prudkin L, et al: Expression of the receptor tyrosine kinase EphA2 is increased in smokers and predicts poor survival in non-small cell lung cancer. Clin Cancer Res 15: 4423-4430, 2009.

26. Wang LF, Fokas E, Juricko J, et al: Increased expression of EphA7 correlates with adverse outcome in primary and recurrent glioblastoma multiforme patients. BMC Cancer 8: 79, 2008.

27. Noren NK and Pasquale EB: Paradoxes of the EphB4 receptor in cancer. Cancer Res 67: 3994-3997, 2007.

28. Korner M and Miller LJ: Alternative splicing of pre-mRNA in cancer: focus on $\mathrm{G}$ protein-coupled peptide hormone receptors. Am J Pathol 175: 461-472, 2009. 